\title{
To Preserve or Not to Preserve the Orbit in Paranasal Sinus Neoplasms: A Meta-Analysis
}

\author{
Camilo Reyes $^{1} \quad$ Eric Mason ${ }^{2,3}$ \\ C. Arturo Solares 2,3 \\ ${ }^{1}$ Department of Otolaryngology, Universidad del Rosario, \\ Bogota, Colombia \\ 2 Department of Otolaryngology, Georgia Regents University, \\ Augusta, Georgia, United States \\ ${ }^{3}$ Center for Skull Base Surgery, Georgia Regents University, Augusta, \\ Georgia, United States \\ ${ }^{4}$ Department of Otolaryngology - Head and Neck Surgery, The Ohio \\ State University Wexner Medical Center, Columbus, Ohio, \\ United States
}

Carrie Bush $^{2}$ Ricardo Carrau ${ }^{4}$

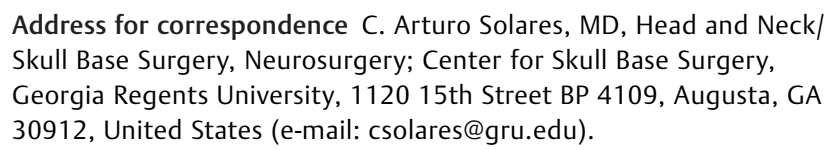
Skull Base Surgery, Neurosurgery; Center for Skull Base Surgery, Georgia Regents University, 1120 15th Street BP 4109, Augusta, GA 30912, United States (e-mail: csolares@gru.edu).

J Neurol Surg B 2015;76:122-128.

\section{Introduction}

Paranasal sinus neoplasms account for 3 to $5 \%$ of all malignant neoplasms of the upper respiratory tract. Estimated incidence in the United States is 0.556 to 1.0 cases per 100,000 population. In Asia and Africa the incidence is higher: 2.5 to 2.6 cases

received

June 30,2014

accepted

July 21, 2014

published online

November 6, 2014
Context The effect on survival of orbital evisceration on patients with paranasal sinus neoplasms has not been well established.

Objective To review systematically the available literature concerning survival in patients who undergo surgery for paranasal sinus neoplasm with and without preservation of the eye.

Data Source A retrospective meta-analysis of English and non-English articles using Medline and the Cochrane database.

Eligibility Criteria Studies analyzing 5-year survival rates in patients who had orbital evisceration compared with orbital preservation for the treatment of paranasal sinus neoplasms were included in the final analysis.

Data Extraction Independent review by two authors using predefined data fields.

Data Synthesis A meta-analysis of four articles involving 443 patients was performed using the DerSimonian-Laird random-effects method.

Results Our analysis revealed a total effect size of 0.964 in favor of preservation of the eye; however, these results are not robust, having a true effect size anywhere from 0.785 to 1.142 with a $95 \%$ confidence interval.

Limitations Only retrospective observational studies were included because a prospective randomized study cannot be performed in this population.

Conclusion Our study supports the notion that in select patients preservation of the eye may yield a different outcome when compared with orbital evisceration.

per 100,000 population. ${ }^{1}$ Due to the contiguity of the nasal and paranasal sinuses, identifying the specific site of origin is difficult; therefore, malignant tumors of the nasal cavity are often clustered with those of the paranasal sinuses.

Compromise of the dura mater, brain, or the soft tissues of the orbit (including orbital periosteum) has a negative

(c) 2015 Georg Thieme Verlag KG Stuttgart · New York
DOI http://dx.doi.org/ 10.1055/s-0034-1390403. ISSN 2193-6331. 
influence over oncologic and functional outcomes of patients with paranasal sinus cancers, and some have suggested that there should be no hesitancy in removing seemingly involved tissues (macroscopic). Involvement of the orbital soft tissue is an independent factor significantly influencing survival. ${ }^{2}$ However, the impact of orbital evisceration or preservation on survival is still not clear. The incidence of orbital invasion depends mainly on the site of origin and the histology of the malignancy. Because of these variables, indications to eviscerate the orbit have long been and continue to be controversial. This has been a source of confusion when reporting guidelines and outcomes, making it a challenge in developing an evidence-based approach. Several authors and institutions have specific criteria to manage the orbit and its contents when paranasal sinus neoplasms compromise them. However, most authors consider that survival rates after orbital preservation, as opposed to orbital clearance (preservation only of the lids and palpebral conjunctiva) or orbital evisceration (complete removal of the contents of the orbit), are not significantly different.

The purpose of this study was to review the available literature systematically and thus provide a basis for updated recommendations regarding the standards of practice for preservation or clearance/evisceration of the orbit in patients with paranasal sinus neoplasms.

\section{Objective}

To examine the impact of orbital evisceration on survival in patients with paranasal sinus neoplasm, we reviewed retrospective observational studies that compared 5-year survival rates in patients with paranasal sinus neoplasms who underwent orbital evisceration or orbital preservation.

\section{Methods}

To identify eligible studies, we searched (Medline and Cochrane Central through Ovid Interface) from 1978 through October 2013 using Medical Subject Headings (MeSH) terms Orbit Evisceration AND Paranasal sinus neoplasms mortality, pathology, surgery, therapy, radiotherapy and/or epidemiology. We also performed a second search with MeSH terms Orbit Evisceration AND Head and Neck Neoplasms epidemiology, etiology, surgery, radiotherapy and/or therapy.

Eligibility of the manuscripts was predicated on a description of the orbital management (preservation or other) in patients of any age with paranasal sinus neoplasms and the inclusion of 5-year survival rate. No publication date or publication status restrictions were imposed. Patients with benign tumors of the paranasal sinus were excluded. English- and Spanish-language manuscripts were acceptable for the analysis. It was decided a priori to exclude publications on functional outcomes, aesthetic sequelae, and reconstruction procedures because these issues were beyond the scope of the review.

\section{Study Selection}

Eligibility assessment was preformed independently by two reviewers (C.R. and E.M.) who screened all the titles and abstracts for candidate studies, and full-text publications for eligibility. Disagreement between reviewers was resolved by consensus.

\section{Data Collection}

Using a standardized form, the following data were included from all eligible studies:

- Descriptive data: Information was extracted from each included article on sample size, mean patient age, percentage of male and female gender, length of follow-up, histopathology diagnosis, location of tumor, type of orbital invasion, evisceration or preservation of the orbit, and 5 year-survival.

- Methodologic data: Method to estimate 5-year survival.

- Outcome data: 5-year survival of orbit-evisceration and orbit-preservation cohorts.

Effect sizes were calculated by risk ratios. These risk ratios were weighed according to their variance in each particular study.

To explore the variability in study results, we specified the following hypothesis before conducting the analysis. The null hypothesis for the Cochran $Q$ test is that there are no differences between the variables. ${ }^{3}$ If the calculated probability is low ( $p$ less than the selected significance level) the null hypothesis is rejected, and it can be concluded that the proportions in at least two of the variables are significantly different from each other.

Measures of variability in study results (heterogeneity) were calculated to assess the consistency of the results. The Cochrane $Q$ test is computed by summing the squared deviations of each study's estimate from the overall meta-analytic estimate. A $p$ value is obtained by comparing the statistic with a chi-square distribution with $k-1$ degrees of freedom (where $k$ is the number of studies).

To assess the chances of survival after orbit evisceration relative to orbit preservation, the effect size was measured using a risk ratio. The risk ratio measures the percentage of 5-year survival generated after orbital evisceration over the percentage of 5-year survivals that preservation generated. Hence the risk ratio is calculated by:

$$
r=\frac{P_{e x}}{P_{p r}}, 0 \leq r \leq \infty
$$

Where $P_{\mathrm{ex}}$ is the ratio between the number of patients that had a 5-year survival through orbital evisceration and the total number of eviscerations. $P_{\mathrm{pr}}$ is the ratio between the number of 5-year survivals through orbital preservation and the total number of preserved orbits. This ratio was calculated for the studies selected in the data collection process. Note that an effect size $>1$ indicates that the 5 -year survival rate after orbital evisceration is greater than that after orbital preservation. Inversely, an effect size $<1$ suggests that the 5 -year survival rate after orbital preservation is greater than that after evisceration. Finally, an effect size of 1 indicates no difference between the 5-year survival rates.

\section{Planned Methods of Analysis}

The total effect, overall intervals (confidence interval [CI]), and weights were calculated using the DerSimonian-Laird 
random-effects method. Because the studies selected allowed for variation in fixed attributes such as age and gender, the model selected allowed for heterogeneity between the studies. Hence it is assumed that the true effect is different for each study. We used the $I^{2}$ test to estimate the percentage of total variation across studies that is due to heterogeneity rather than chance.

We could not assess the possibility of publication bias by evaluation with a funnel plot because of the small number of studies that address this issue and the fact that only retrospective studies were available for this analysis. No articles that assessed 5-year survival in patients with paranasal sinus cancer who had orbital preservation or other treatment, despite their results, were excluded.

\section{Results}

Four articles were identified for inclusion in the review. The search of Medline and Cochrane databases identified a total of 89 citations. After adjusting for duplicates, 60 remained. Of these, 40 studies were discarded because after reviewing the abstracts it appeared these articles clearly did not meet the inclusion criteria. Two additional studies were discarded because a full text of the study was not available. Full text of the remaining 18 citations was examined in more detail. It appeared that 12 studies did not meet the inclusion criteria as previously described. Five articles were excluded because they did not estimate 5-year survival between patients who had orbital evisceration or preservation, ${ }^{4-8}$ five more because they independently assessed patients who had orbital evisceration or preservation, ${ }^{6,9-12}$ one study assessed reconstructive and aesthetic consequences of orbital evisceration, ${ }^{13}$ and one analyzed the effects of chemotherapy for organ preservation in patients with paranasal sinus neoplasms. ${ }^{14}$ An article by Som ${ }^{15}$ was inaccessible. The Perry et al $^{16}$ article was excluded because we found several flaws in the population analysis. It gave information that was difficult to interpret, not all results were analyzed, and it did not mention the 5-year survival rate between both groups, despite the fact that it is cited by other authors (-Fig. 1).

All four articles included here are retrospective studies of patients with paranasal sinus neoplasm with involvement of the orbit who had craniofacial resection with orbital evisceration or preservation. The included studies involved 443 patients. Men were more frequently affected by paranasal sinus neoplasms in all studies. The age of presentation was broad, ranging from 18 to 83 years ( - Table $\mathbf{1}$ ). The ethmoid sinus and surrounding structures (anthroethmoid, orbitoethmoid) was the most common location of the tumor. Concomitant involvement of the nasal cavity and the maxillary sinus was frequent. The frontal sinus and nasopharyngeal tumors were uncommon. The two most frequent malignancies were squamous cell carcinomas (SCCs) and adenocarcinomas. With the exception of the study from Wu et $\mathrm{al}^{17}$ (only SCC), all

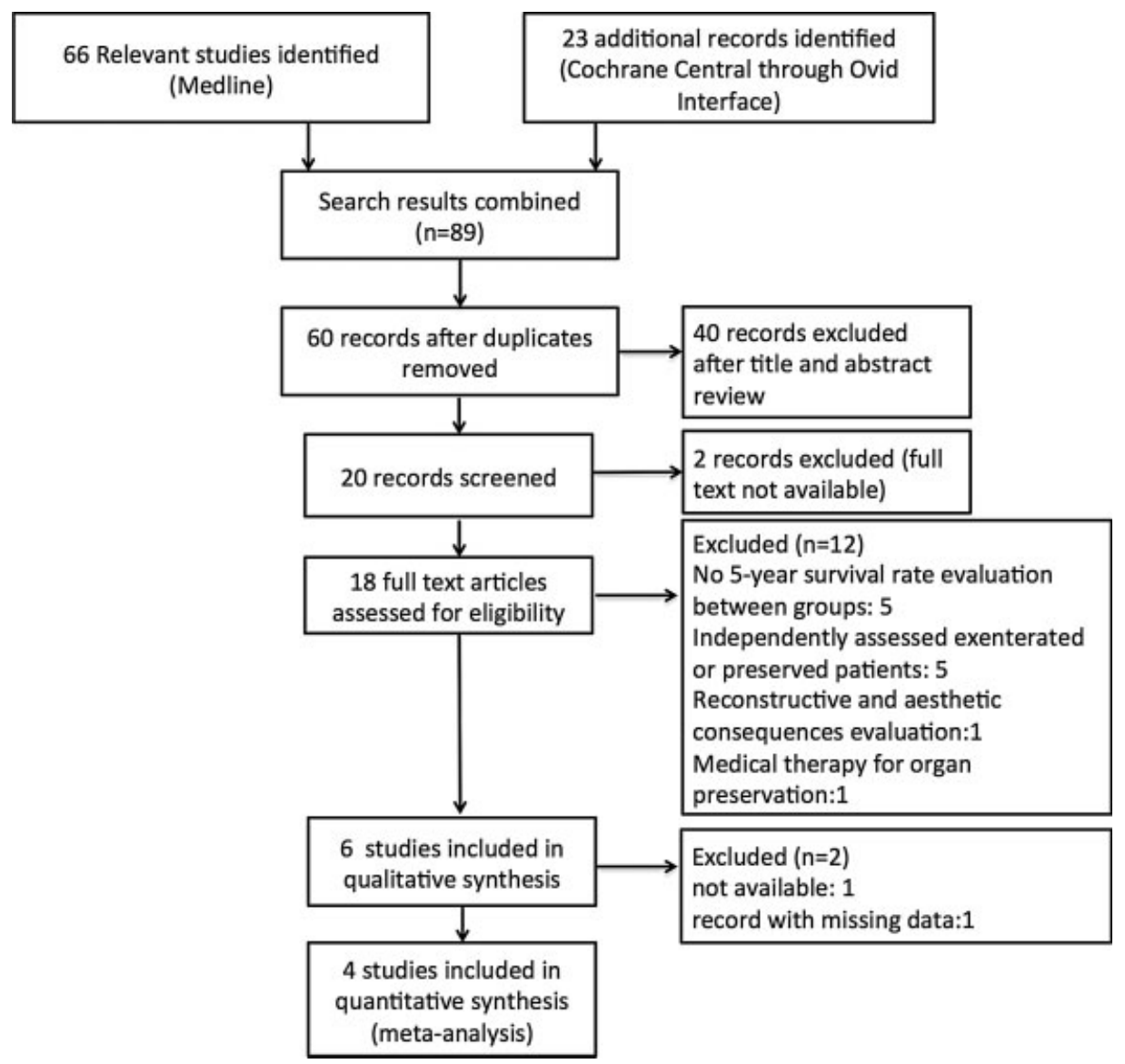

Fig. 1 Summary of systematic literature review. 
Table 1 Summary of included studies evaluating 5-year survival in patients with paranasal sinus cancer who had orbital preservation/evisceration

\begin{tabular}{|c|c|c|c|c|c|c|c|}
\hline Study & No. of patients & Male & Female & Age, y & Orbital invasion & Orbital evisceration & Orbital preservation \\
\hline Wu et $a l^{17, a}$ & 139 & 94 & 45 & $19-74$ & 111 & 88 & 23 \\
\hline Lund et $\mathrm{al}^{19, \mathrm{~b}}$ & 209 & 143 & 66 & $7-77$ & 64 & 41 & 23 \\
\hline Imola et $\mathrm{al}^{20}$ & 66 & 41 & 25 & $18-83$ & 66 & 12 & 54 \\
\hline lannetti et al ${ }^{18, c}$ & 29 & 18 & 11 & $22-76$ & 24 & 9 & 13 \\
\hline
\end{tabular}

${ }^{a}$ Only patients with squamous cell carcinoma.

b Patients were young in this study because it included patients with benign and malignant disease. Only patients with malignant disease were included in the analysis.

${ }^{\mathrm{c}}$ Four patients were excluded from the final analysis because of very advanced disease.

studies included patients with different histologic types of paranasal sinus neoplasms (-Table 2). Grade or anatomical compromise by tumor was described as an indication for orbital evisceration by all authors. Indications for evisceration were included for each author according to the three grades of orbital invasion suggested by lannetti et al. ${ }^{18}$ Interventions received were craniofacial resection with orbital preservation or evisceration, preoperative or postoperative radiotherapy, and chemotherapy. The primary outcome assessed was 5 -year survival in all studies (-Table 3 ). Follow-up time was broad; Wu et al $^{17}$ did not mention a follow-up period; for Lund et $\mathrm{al}^{19}$ it is assumed to be for at least 17 years, for Imola et $\mathrm{al}^{20}$ it was 2 to 10.5 years, and for Ianneti ${ }^{18}$ et al it was 11 months to 17 years (mean: 75 months).
A statistical test of heterogeneity revealed that results across studies were not consisten (-Table 4). An analysis of individual studies was made using the DerSimonian-Laird random-effects method (- Table 5 ).

Five-year survival was available for all four studies. Only one study reported the number of deaths; however, these were from complications and not from the disease. Effect sizes are calculated by risk ratios. These risk ratios are weighed according to their variance in each particular study. Three of the four studies have effect sizes very close to 1 . Wu et $\mathrm{al}^{17}$ is the only study that finds evidence, both in the effect size and the $\mathrm{CI}$, in favor of preservation giving higher odds of a 5 -year survival. Although Imola et $\mathrm{al}^{20}$ included 66 patients, they only analyzed 5-year survival in two groups of patients

Table 2 Anatomical tumor location and pathology of included studies

\begin{tabular}{|c|c|c|c|c|c|}
\hline \multirow{2}{*}{$\begin{array}{l}\text { Source } \\
\text { Wu et al }{ }^{17}\end{array}$} & \multirow{2}{*}{$\begin{array}{l}\text { Site } \\
\text { Not mentioned }\end{array}$} & \multicolumn{4}{|l|}{ Pathology } \\
\hline & & SCC & 139 & & \\
\hline \multirow[t]{10}{*}{ Lund et al ${ }^{19}$} & \multirow{10}{*}{$\begin{array}{l}\text { Ethmoid (90) } \\
\text { Nasal cavity (53) } \\
\text { Anthroethmoid (28) } \\
\text { Orbitoethmoid (18) } \\
\text { Sphenoid (9) } \\
\text { Frontal(6) } \\
\text { Frontoethmoid (3) } \\
\text { Frontoethmoid/maxilla (2) }\end{array}$} & Adenocarcinoma & 42 & Olfactory neuroblastoma & 26 \\
\hline & & SCC & 25 & Chondrosarcoma & 19 \\
\hline & & Adenoid cystic & 15 & Anaplastic & 10 \\
\hline & & Malignant melanoma & 8 & Cylindric cell carcinoma & 5 \\
\hline & & Rhabdomyosarcoma & 3 & Metastasis & 3 \\
\hline & & Malignant histiocytoma & 2 & Spindle cell sarcoma & 1 \\
\hline & & Carcinosarcoma & 1 & Osteogenic sarcoma & 1 \\
\hline & & Angiosarcoma & 1 & Hemangiopericytoma & 1 \\
\hline & & Alveolar soft part sarcoma & 1 & Malignant schwannoma & 1 \\
\hline & & Mucoepidermoid carcinoma & 1 & Ewing sarcoma & 1 \\
\hline \multirow[t]{3}{*}{ Imola et al ${ }^{a}$} & \multirow{3}{*}{$\begin{array}{l}\text { Ethmoid (13) } \\
\text { Maxillary (32) } \\
\text { Nose (13) } \\
\text { Nasopharynx (8) }\end{array}$} & SCC & 24 & Adenomatous Carcinoma & 13 \\
\hline & & SNUC & 8 & Sarcoma & 12 \\
\hline & & Other & 9 & & \\
\hline \multirow[t]{4}{*}{ lannetti et al ${ }^{18,20}$} & \multirow{4}{*}{$\begin{array}{l}\text { Ethmoids (24) } \\
\text { Nasal cavities (20) } \\
\text { Maxillary (15) } \\
\text { Sphenoidal (11) } \\
\text { Frontal (5) }\end{array}$} & Adenocarcinoma & 7 & SCC & 6 \\
\hline & & Adenoid-cystic carcinoma & 4 & Undifferentiated carcinoma & 3 \\
\hline & & Sarcoma & 2 & Adenosquamous carcinoma & 1 \\
\hline & & Olfactory neuroblastoma & 1 & & \\
\hline
\end{tabular}

Abbreviations: SCC, squamous cell carcinoma; SNUC, sinonasal undifferentiated carcinoma.

${ }^{a}$ Only patients with malignant neoplasms were included in the analysis (malignant neoplasm $n=167$ [68\%]).

Note: The two most common neoplasms were SCC and adenocarcinoma. 
Table 3 Indications for evisceration and 5-year survival in both groups

\begin{tabular}{|l|l|l|l|}
\hline Source & Indication for evisceration & $\begin{array}{l}\text { 5-year survival } \\
\text { evisceration, \% }\end{array}$ & $\begin{array}{l}\text { 5-year survival } \\
\text { preservation, \% }\end{array}$ \\
\hline Wu et al $^{17}$ & Invasion medial orbital wall & 34.8 \\
\hline Lund et al $^{19}$ & Transgression of the periosteum & \\
\hline Imola et al $^{20}$ & $\begin{array}{l}\text { Orbital fat involvement, } \\
\text { extraocular muscle invasion, } \\
\text { orbital apex, or eyelid invasionc }\end{array}$ & 27.3 & 26 \\
\hline lannetti et al $^{18}$ & $\begin{array}{l}\text { Invasion of the medial rectus } \\
\text { muscle, optic nerve, ocular bulb, } \\
\text { or the skin overlying the eyelid }\end{array}$ & 46 & 53 \\
\hline
\end{tabular}

${ }^{a}$ Wu et al used grade I as an indication for evisceration.

bLund et al used grade II.

Imola and lanneti used grade III as an indication of evisceration.

Table 4 Heterogeneity test from random effect model

\begin{tabular}{|l|l|l|}
\hline Test & Value & $P$ \\
\hline Cochrane $Q$ & 17.4 & 0.002 \\
\hline$P^{2}(\%)$ & 77.01 & \\
\hline
\end{tabular}

Note: Statistical test of heterogeneity reveal that results across studies were not consistently the same. Results of the Cochran $Q$ test revealed the presence of heterogeneity indicated by the low $p$ value $(0.002)$. Our estimates indicate that $77.01 \%\left(I^{2}\right)$ of total variation across studies is due to heterogeneity.

(patients with SCC (24 of 66) and adenomatous nonepidermoid carcinoma (13 of 66) who had preserved or eviscerated orbits according to their criteria (-Table 5 ).

The total effect size from the four studies is 0.964 , indicating that preservation gives a slightly better chance for 5-year survival over evisceration. However, the 95\% CI includes values in a range between 0.785 and 1.142 , which indicates that the true effect size could be 1 or $>1$. - Fig. 2 illustrates the forest plot for the DerSimonian-Laird random-effects results.

\section{Discussion}

Overall, the evidence is not sufficiently robust to determine if orbital preservation or evisceration, as part of craniofacial resection, has a better 5-year survival rate in patients with paranasal sinus neoplasm. However, some acceptable evidence suggests that preservation of the eye gives a better chance for 5-year survival over evisceration (total effect: 0.964; CI > 95\%, 0.785-1.142), an effect that may be limited to patients with SCC and adenocarcinoma because these two where the most common neoplasms.

We aimed to identify articles in which 5-year survival rate was assessed. All of the data are drawn from retrospective studies due to the obvious ethical principles of performing a randomized control trial in this population.

The issue of when to eviscerate the orbit generates vigorous debate. However, there are little objective data to guide the head and neck surgeon. It seems that in recent times, when quality of life plays a pivotal role in the treatment of cancer, indications to eviscerate the orbit are more stringent and include primarily invasion of the rectus muscle, ocular bulb, optic nerve, and/or the overlying skin.

Iannetti et $\mathrm{al}^{18}$ defined three stages of orbital invasion: grade I, erosion or destruction of the medial orbital wall; grade II, extraconal invasion of the periorbital fat; and grade III, invasion of the medial rectus muscle, optic nerve, ocular bulb, or the skin overlying the eyelid. Despite the suggested indications to eviscerate the orbit, there are no universally accepted criteria. Grade III orbital invasion is the most accepted indication because it has a more conservative indication on eye structures. This point was addressed by

Table 5 Effect sizes and DerSimonian-Laird random-effects results for individual studies

\begin{tabular}{|c|c|c|c|c|}
\hline Study & Risk ratio & 95\% lower limit & 95\% upper limit & Weights \\
\hline Wu et al $^{17}$ & 0.78 & 0.692 & 0.868 & 16.74 \\
\hline Lund et al $^{19}$ & 1.12 & 0.982 & 1.258 & 16.71 \\
\hline Imola et $\mathrm{al}^{20}$ & 0.96 & 0.827 & 1.093 & 16.71 \\
\hline  & 1.05 & 0.737 & 1.363 & 16.51 \\
\hline Total & 0.964 & 0.785 & 1.142 & 100 \\
\hline
\end{tabular}

Note: Summary of the risk ratio (effect size) for the four studies. Weights are proportional to a study's variance and sample size. Hence larger weights were given to studies with small variance, and small weights to those with large variance, generally corresponding to smaller sample sizes. 


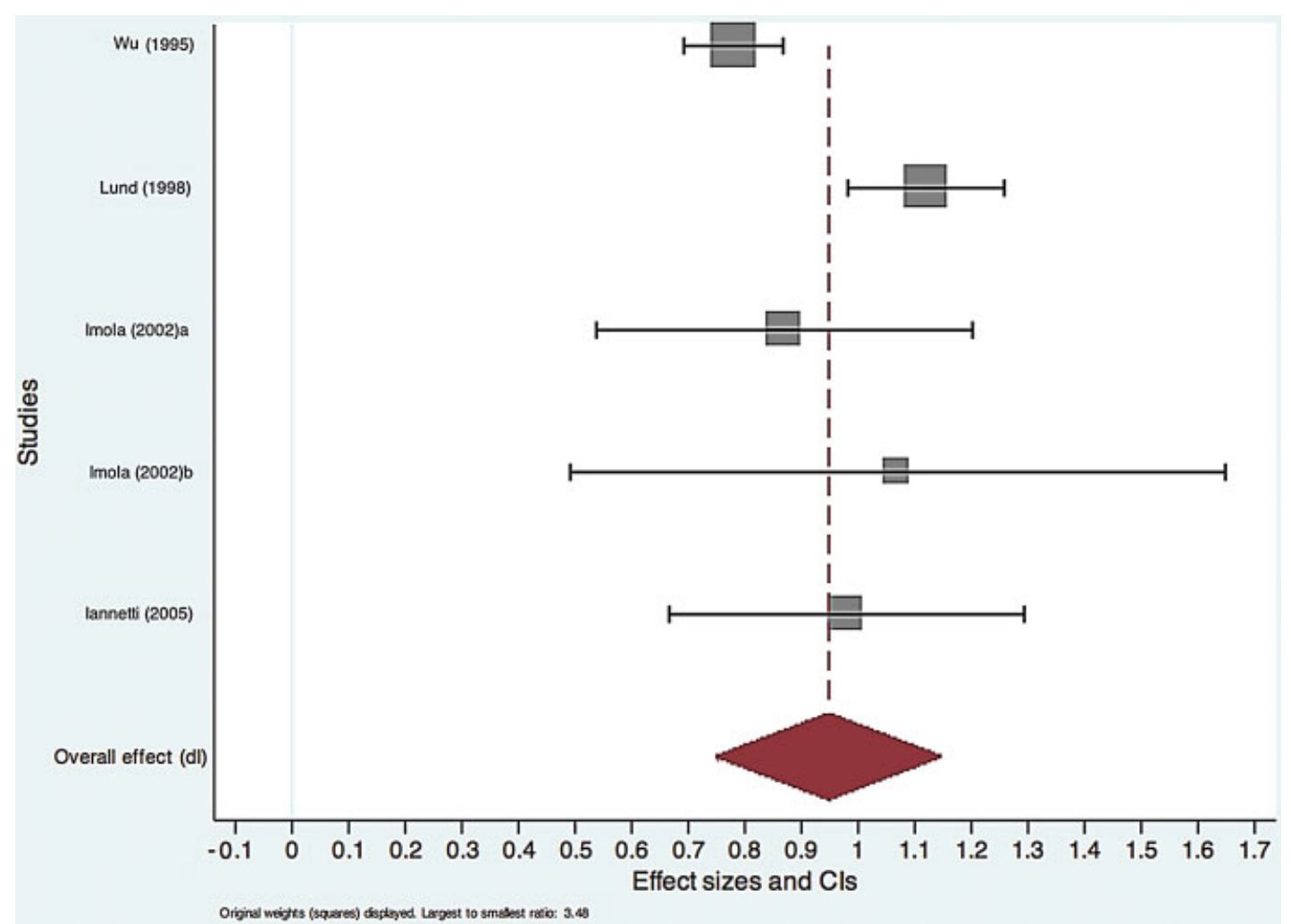

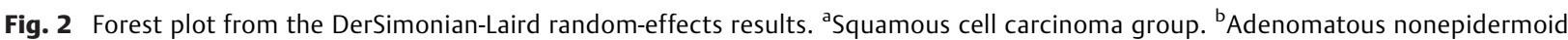
carcinoma group. $\mathrm{Cl}$, confidence interval.

Wu et al. ${ }^{17}$ They noticed that in 62 of the 65 pathologies analyzed (95\%), invasion of the orbit was limited and the orbit could have been preserved by resecting the periorbita. Also, preservation of the orbit was not associated with a significantly higher rate of local recurrence $(12.5 \%$ in eviscerated versus $8.6 \%$ in preserved). However, this is only valid for SCC. These results are similar to those found by Carrau et al, ${ }^{21}$ who also retrospectively studied patients with SCC.

Lund et $\mathrm{al}^{19}$ concluded that preservation of the orbit does not affect survival significantly (5-year survival of $26 \%$ preserved versus $29 \%$ eviscerated). Imola et $\mathrm{al}^{20}$ considered grade III as an indication for orbital evisceration; however, they tried to be as conservative as possible by using microscopically assisted dissection to determine whether orbital preservation was feasible. As an indication of evisceration, they considered penetration beyond the periorbita into orbital fat that cannot be safely resected or when invasion into extraocular eye muscles was present. Iannetti et $\mathrm{al}^{18}$ also considered grade III as an indication for orbital evisceration. Like other authors they included several histologic types. They noticed no difference between orbital evisceration versus preservation. Nonetheless, their $\mathrm{Cl}$ is the widest in all four studies (0.737-1.363), making these results less reliable. It is important to mention that in three studies, except for $\mathrm{Wu}$ et al, ${ }^{17}$ all patients received postoperative radiotherapy, which plays an important role in disease recurrence and survival; however, such debate is beyond the scope of this review.
The meta-analysis reported here combines data across studies to estimate treatment effects with more precision than is possible in a single study. The main limitation of this meta-analysis, as with any overview, is that paranasal sinus neoplasms are a relatively rare disease with $\sim 70$ histologic subtypes; therefore, it is very difficult to cluster all paranasal sinus cancer as just one entity. Also, the lack of clinical guidelines leads to diversity in management, which makes outcomes-based studies difficult to interpret. The variability in indications for evisceration makes it difficult to analyze the data because it is conceivable that evisceration could have been avoided in patients who may not have had severe compromise. Thus data from studies with more aggressive indications for evisceration may skew the results in favor of evisceration having a positive impact on survival. Alternatively, patients having undergone evisceration may represent a more aggressive subpopulation, thus skewing the data in favor of orbital preservation.

\section{Conclusion}

The role of orbital evisceration still needs to be clarified, and the optimal indications for it are not certain. Over time, indications for evisceration have changed; as of now, a significant number of physicians do their best to preserve the orbit. Despite attempts to stratify results based on tumor stage and degree of orbital invasion, a selection bias exists in all of these studies because the tumors in more advanced 
stages (orbital apex invasion) with expected worse outcomes were treated with orbital clearance, whereas those with more favorable orbital extension were treated with more conservative approaches.

Our statistical analysis suggests there is no strong evidence favoring either evisceration or preservation of the eye in patients with orbital compromise by paranasal sinus neoplasms. However, in select patients with specific paranasal sinus neoplasm, preservation of the eye might yield a significantly different outcome. The 5-year survival rates after preservation are promising, but the data should be analyzed with caution. Tumor histology plays a significant independent role in patient outcome, irrespective of orbital invasion. Nevertheless, the histologic diversity of tumor types in this region confounds accurate statistical analysis.

Our study has the limitation that the effect size could be 1 or $>1(0.97, \mathrm{CI}>95 \%, 0.785-1.114)$, which means the question still needs to be answered and no absolute parameters can be assigned to one management or the other. Given the increased interest in improving quality of life following cancer care, and specifically orbital preservation in patients with paranasal sinus neoplasms, a prospective multi-institutional study reporting 5-year survival rates in both cohorts (eviscerated and preserved), with further analysis by gender, age, histologic type, and degree of orbital compromise, is a must.

\section{Acknowledgments}

The authors would like to acknowledge the contribution of Dr. Juan Alejandro Gelves for his pivotal role in the statistical analysis of this study.

\section{References}

1 Haerle SK, Gullane PJ, Witterick IJ, Zweifel C, Gentili F. Sinonasal carcinomas: epidemiology, pathology, and management. Neurosurg Clin N Am 2013;24(1):39-49

2 Suarez C, Llorente JL, Fernandez De Leon R, Maseda E, Lopez A. Prognostic factors in sinonasal tumors involving the anterior skull base. Head Neck 2004;26(2):136-144

3 Sheskin DJ. Handbook of Parametric and Nonparametric Statistical Procedures. 5th ed. Boca Raton, FL: Chapman \& Hall/CRC Taylor \& Francis Group; 2011

4 Stern SJ, Goepfert H, Clayman G, Byers R, Wolf P. Orbital preservation in maxillectomy. Otolaryngol Head Neck Surg 1993;109(1): $111-115$
5 Shah JP, Kraus DH, Bilsky MH, Gutin PH, Harrison LH, Strong EW. Craniofacial resection for malignant tumors involving the anterior skull base. Arch Otolaryngol Head Neck Surg 1997;123(12): 1312-1317

6 Nishino H, Ichimura K, Tanaka H, et al. Results of orbital preservation for advanced malignant maxillary sinus tumors. Laryngoscope 2003;113(6):1064-1069

7 Essig GF, Newman SA, Levine PA. Sparing the eye in craniofacial surgery for superior nasal vault malignant neoplasms: analysis of benefit. Arch Facial Plast Surg 2007;9(6):406-411

8 Rajapurkar M, Thankappan K, Sampathirao LMCS, Kuriakose MA, Iyer S. Oncologic and functional outcome of the preserved eye in malignant sinonasal tumors. Head Neck 2013;35(10):1379-1384

9 McCary WS, Levine PA, Cantrell RW. Preservation of the eye in the treatment of sinonasal malignant neoplasms with orbital involvement. A confirmation of the original treatise. Arch Otolaryngol Head Neck Surg 1996;122(6):657-659

10 Nazar G, Rodrigo JP, Llorente JL, Baragaño L, Suárez C. Prognostic factors of maxillary sinus malignancies. Am J Rhinol 2004;18(4): 233-238

11 Howard DJ, Lund VJ, Wei WI. Craniofacial resection for tumors of the nasal cavity and paranasal sinuses: a 25-year experience. Head Neck 2006;28(10):867-873

12 Kuo C-H, Gao K, Clifford A, Shannon K, Clark J. Orbital exenterations: an 18-year experience from a single head and neck unit. ANZ J Surg 2011;81(5):326-330

13 Tiwari R, van der Wal J, van der Waal I, Snow G. Studies of the anatomy and pathology of the orbit in carcinoma of the maxillary sinus and their impact on preservation of the eye in maxillectomy. Head Neck 1998;20(3):193-196

14 Papadimitrakopoulou VA, Ginsberg LE, Garden AS, et al. Intraarterial cisplatin with intravenous paclitaxel and ifosfamide as an organ-preservation approach in patients with paranasal sinus carcinoma. Cancer 2003;98(10):2214-2223

15 Som ML. Surgical management of carcinoma of the maxilla. Arch Otolaryngol 1974;99(4):270-273

16 Perry C, Levine PA, Williamson BR, Cantrell RW. Preservation of the eye in paranasal sinus cancer surgery. Arch Otolaryngol Head Neck Surg 1988;114(6):632-634

17 Wu X, Tang P, Qi Y. Management of the orbital contents in radical surgery for squamous cell carcinoma of the maxillary sinus. Chin Med J (Engl) 1995;108(2):123-125

18 Iannetti G, Valentini V, Rinna C, Ventucci E, Marianetti TM. Ethmoido-orbital tumors: our experience. J Craniofac Surg 2005; 16(6):1085-1091

19 Lund VJ, Howard DJ, Wei WI, Cheesman AD. Craniofacial resection for tumors of the nasal cavity and paranasal sinuses-a 17-year experience. Head Neck 1998;20(2):97-105

20 Imola MJ, Schramm VL Jr. Orbital preservation in surgical management of sinonasal malignancy. Laryngoscope 2002;112(8 Pt 1): 1357-1365

21 Carrau RL, Segas J, Nuss DW, et al. Squamous cell carcinoma of the sinonasal tract invading the orbit. Laryngoscope 1999;109(2 Pt 1): 230-235 